\title{
Wernicke's encephalopathy manifesting with diplopia after ileojejunostomy: report of a pediatric case with Hirschsprung disease
}

\author{
Beril Dilber $^{1 \oplus}$, Tülay Kamaşak ${ }^{1 \oplus}$, İlker Eyüboğlu ${ }^{2 \oplus}$, Mehmet Kola $^{3 \oplus}$, \\ Ahmet Taner Uysal ${ }^{3 \oplus}$, Haluk Saruhan ${ }^{4 \oplus}$, Sonay Yalçın ${ }^{4 \oplus}$, Elif Sağ ${ }^{5 \oplus}$, Ali Cansu ${ }^{1 \oplus}$ \\ Departments of ${ }^{1}$ Pediatric Neurology, ${ }^{2}$ Radiology, ${ }^{3}$ Ophthalmology, ${ }^{4}$ Pediatric Surgery and ${ }^{5}$ Pediatric Gastroenterology, Karadeniz \\ Technical University Faculty of Medicine, Trabzon, Turkey.
}

\begin{abstract}
Background. Wernicke's encephalopathy (WE) is a coenzyme-induced disease with acute neuropsychiatric symptoms leading to high mortality and morbidity due to thiamine deficiency. WE is mostly caused by alcoholism in adult populations; however, it is often associated with gastrointestinal surgical procedures, recurrent vomiting, chronic diarrhea, cancer and chemotherapy treatment, systemic diseases, drugs, magnesium deficiency, and malnutrition in children. Although these predisposing factors are considered to be uncommon in children, they are actually highly frequent and can be fatal if not treated promptly.

Case. In this report, we present a patient who developed diplopia during total parenteral nutrition following surgical resection and was diagnosed with WE. The findings of the patient's cranial magnetic resonance imaging (MRI) findings were consistent with those of $\mathrm{WE}$ and the ocular findings of the patient resolved completely after thiamine treatment.
\end{abstract}

Conclusion. Although WE is rare in children it can be prevented by early diagnosis and treatment and oculomotor findings such as diplopia can be a warning sign.

Key words: Wernicke's encephalopathy, thiamine deficiency, diplopia, ileojejunostomy.

Wernicke's encephalopathy (WE) is an acute neurological condition first described by Carl Wernicke, characterized by mental state changes, ocular abnormalities, and cerebellar dysfunction. ${ }^{1}$ Although WE is mostly caused by alcoholism in adult populations, it is often associated with gastrointestinal surgical procedures, recurrent vomiting, chronic diarrhea, cancer and chemotherapy treatment, systemic diseases, drugs, magnesium deficiency, and malnutrition in children..$^{2-5}$ Cranial imaging of WE often indicates symmetrical involvement of the periaqueductal region, the fourth ventricular base, and mammillary body in

\footnotetext{
$\bowtie$ Beril Dilber

beriltem@gmail.com
}

Received 8th October 2019, revised 4th December 2019, 9th December 2019, accepted 15th December 2019. medial thalamus. ${ }^{6-7}$ Mortality associated with WE is $17 \%$ and the symptoms are completely reversible with early diagnosis and treatment. ${ }^{8}$ In this report, we present a patient who developed diplopia during total parenteral nutrition following surgical resection and was diagnosed with WE who then recovered rapidly and completely after thiamine support. By presenting this case, we aimed to emphasize that fatal situations such as WE, which is a rare condition in children, can be prevented by early diagnosis and treatment and that the oculomotor findings of WE can vary considerably, among which diplopia can be a warning sign.

\section{Case Report}

A 12-year-old girl who was being followed for Hirschsprung disease presented to the 
emergency department with a five-day history of frequent vomiting. The patient was diagnosed with paralytic ileus and a jejunoileal resection and bridectomy was performed. A 55$\mathrm{cm}$ section of the small intestine was removed. Total parenteral nutrition was provided to the patient for 2 weeks. On the $23^{\text {rd }}$ day of hospitalization, the patient developed diplopia. On neurological examination, the patient was sleepy and was agitated in the presence of a stimulus, her speech was slow, and she had poor finger-tracking ability, diplopia, and restricted gaze. Visual acuity was 20/60 in the left eye and 20/70 in the right eye. Fundoscopy showed bilateral blurred optic discs and disc hemorrhage (Fig. 1). Deep tendon reflexes were reduced. There was no abnormality on cranial computed tomography (CT) and in blood parameters. On cranial magnetic resonance imaging (MRI), however, T2 and FLAIR sections showed symmetrical hyperintensity in medial thalamus, in the posterior part of the putamen, in the mesencephalon (tectum), in the periaqueductal region, at the level of facial colliculus, and in the mammillary body (Fig. 2-4). Based on these findings, a diagnosis of WE was made and the patient was given two doses of $200 \mathrm{mg} /$ day thiamine support for five days. After the treatment, the visual complaints of the patient resolved completely. Serum thiamine (B1) level was measured by liquid chromatography/mass spectrometry and the

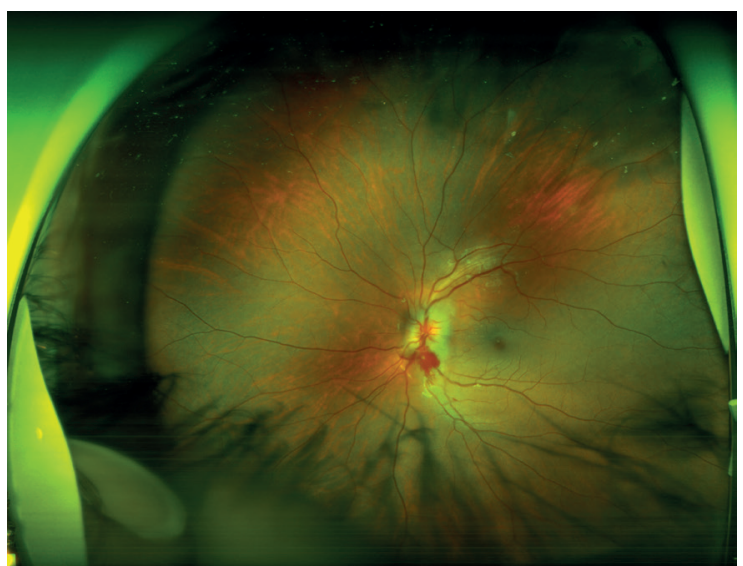

Fig. 1. Fundoscopy showed bilateral blurred optic discs and disc hemorrhage. level measured was found to be lower than the reference levels (9.2 [range, 25-75] nmol/L). A written consent was obtained from the parents.

\section{Discussion}

Wernicke's encephalopathy is a disease characterized by acute neuropsychiatric symptoms as well as high mortality and morbidity associated with thiamine deficiency. ${ }^{9}$ Thiamine is a vitamin essentially required as a coenzyme in the pathways in the brain and is absorbed from the duodenum through active carrier proteins and passes through the blood- brain barrier via active and passive mechanisms, thereby transforming to its active form in glial cells, thiamine pyrophosphate, and participating in the amino acid metabolism of carbohydrates, lipids, and glucose-dependent neurotransmitters.,10,13 The volume loss and cytotoxic edema in astrocytes resulting from thiamine deficiency appears within the first 4 days and leads to endothelial dysfunction and impaired blood-brain barrier function by days 7-10, ultimately resulting in neuronal necrosis and irreversible brain damage by day $14 .{ }^{11-13}$ While the incidence of WE varies across communities, it has been reported to be 0.04$0.013 \%$ in clinical trials and to be higher in autopsy studies $(0.8-2.8 \%)$, with similar rates in children. ${ }^{6,10}$ Typical findings of WE such as mental status change, ophthalmoplegia, gait disturbances, and ataxia are seen in only $16 \%$ of patients. However, WE often manifests with headache and mild drowsiness and progresses gradually. ${ }^{8,11}$ In our patient, diplopia developed in the fourth week after the onset of complaints.

Wernicke's encephalopathy is symmetrical in approximately $50 \%$ of the patients with the involvement of the periductal gray matter, mammillary body, and medial thalamus. Moreover, histopathological changes occur in bilateral dorsal thalamic nuclei in $100 \%$ of the patients. In our patient's MRI, symmetric hyperintensity was detected in medial thalamus, in the posterior part of the putamen, in the mesencephalon (tectum), in the periaqueductal 


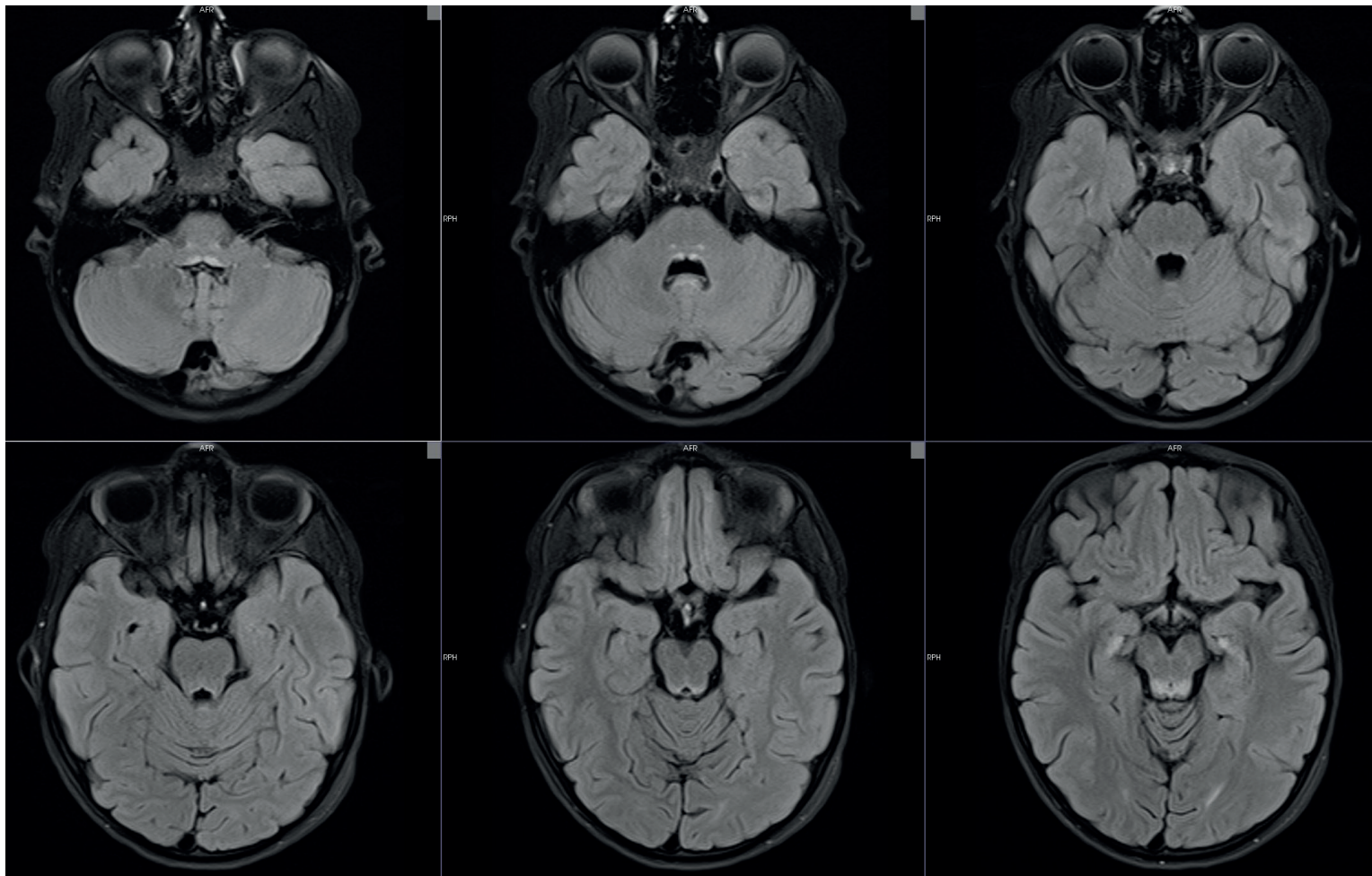

Fig. 2. ADC sections showed symmetrical diffusion restriction in medial thalamus, in the posterior part of the putamen, in the mesencephalon.
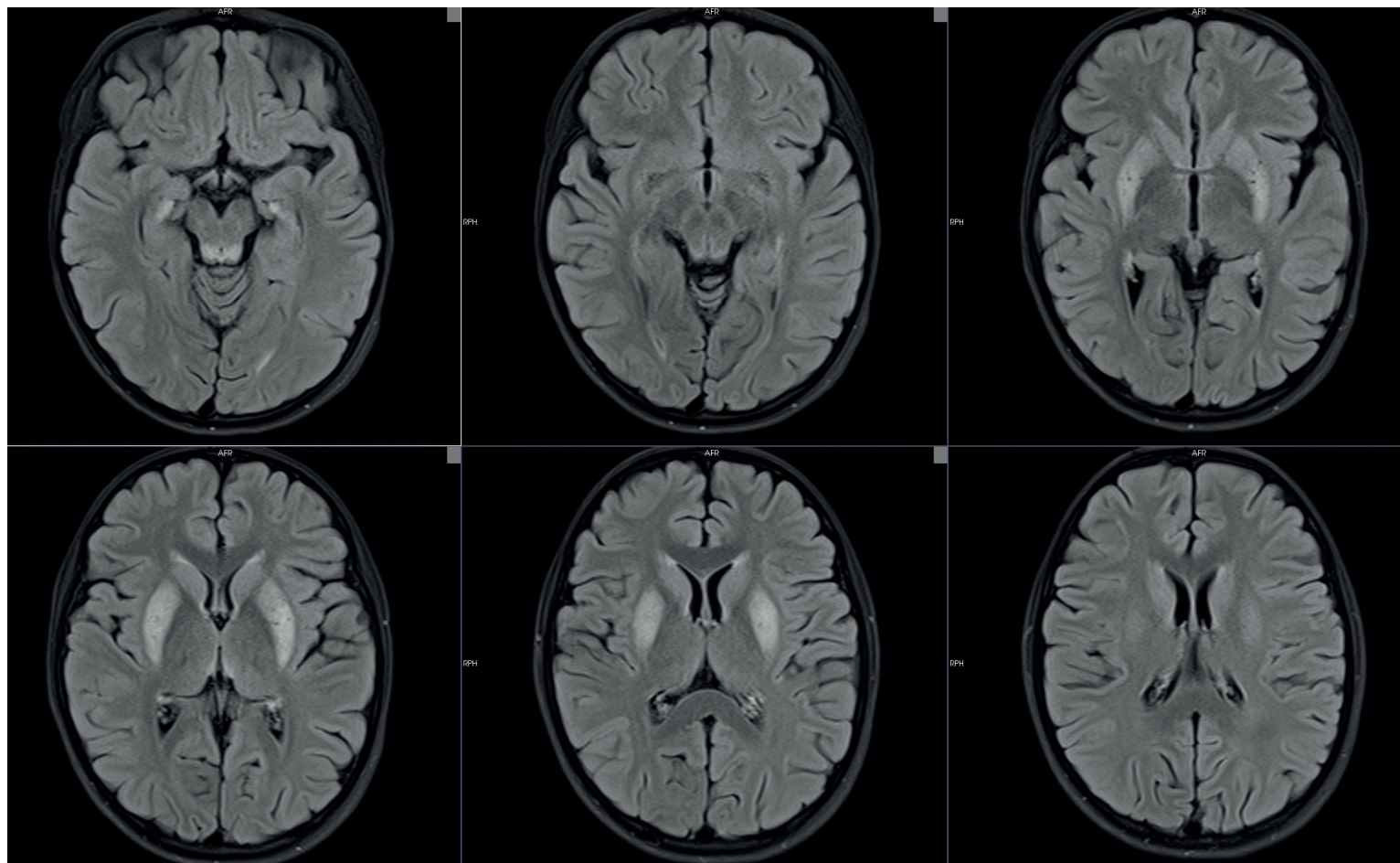

Fig. 3. DWI sections showed symmetrical hyperintensity in medial thalamus, in the posterior part of the putamen, in the mesencephalon (tectum), in the periductal region, at the level of facial colliculus, and in the mammillary body. 

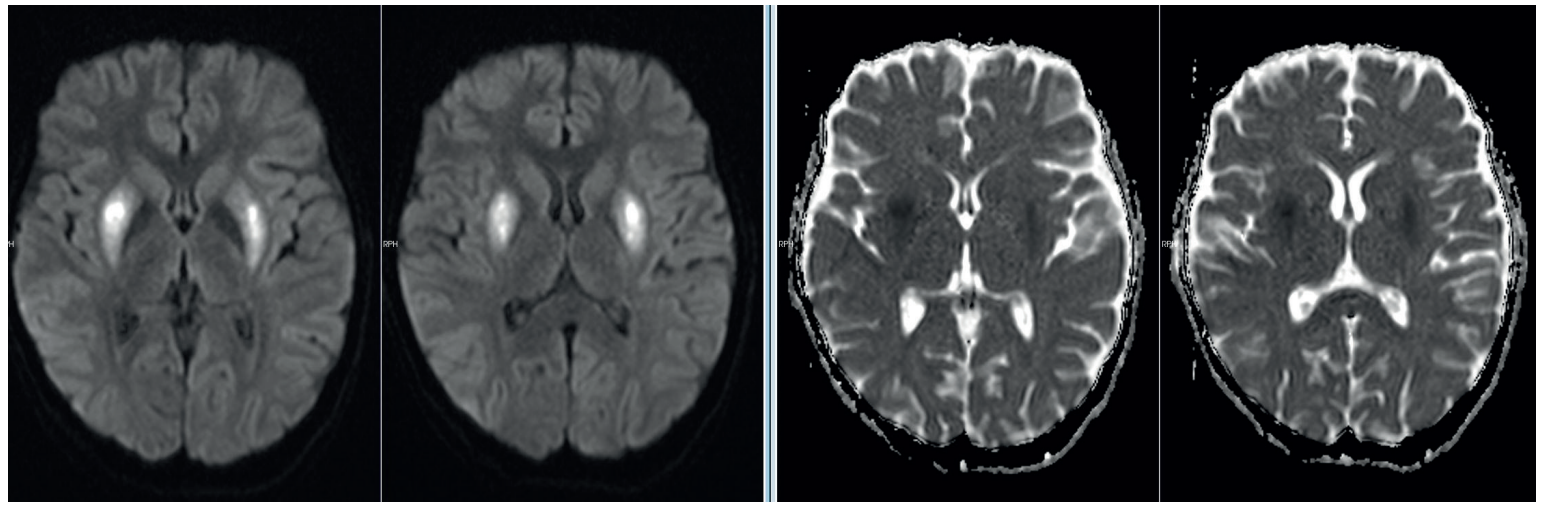

Fig. 4. Flair sections showed symmetrical hyperintensity in medial thalamus, in the posterior part of the putamen, in the mesencephalon (tectum), in the periductal region, at the level of facial colliculus, and in the mammillary body.

region, at the level of facial colliculus, and in the mammillary body. The ocular findings of WE such as disc edema and retinal bleeding, which are often unclear, can present as optic neuritis which is mostly seen in comatose and delayed patients. ${ }^{15}$ However, in our patient, optic disc edema and retinal hemorrhage occurred in a period when the early changes in consciousness were not apparent. The initiation of empirical therapy in WE undergoing surgical intervention can be life-saving. The recommended thiamine dose in children is $100-200 \mathrm{mg}$ /day twice daily or $1.8 \mathrm{mg}$ per kilocalories and $100 \mathrm{mg} /$ day, which is the optimal dosage needed for passing through the blood-brain barrier. ${ }^{16-18}$ Thiamine was given in two doses of $200 \mathrm{mg} /$ day intravenously since the patient was nonalcoholic, and in our patient, diplopia resolved on day 2 and all of the symptoms resolved by day 5 after the initiation of the thiamine therapy.

In conclusion, WE is a clinical condition that should be kept in mind by clinicians when vitamin absorption is prevented in children, particularly in those with predisposing intestinal system diseases such as Hirschsprung disease and those requiring long-term total parenteral nutrition following intestinal surgery. Early diagnosis of WE is highly important as it is a cause of treatable and preventable mortality. In this report, we presented a patient who was receiving postoperative treatment for Hirschsprung disease and was admitted with the complaint of diplopia. The patient received a prompt diagnosis of WE via MRI findings, and was treated completely after thiamine therapy.

\section{REFERENCES}

1. Wernicke C. Die akute haemorrhagische polioencephalitis superior. In: Lehrbuch der Gehirnkrankheiten fur Aerzte und Studirende, Bd II, Kassel: Fisher Verlag, 1881: 229-242.

2. Sechi G, Serra A. Wernicke's encephalopathy: new clinical settings and recent advances in diagnosis and management. Lancet Neurol 2007; 6: 442-455.

3. Kamaşak T, Kul S, Tuşat M, Ozgun N, Cansu A. A case of wernicke encephalopathy developing after ileal bypass surgery. Pediatr Emerg Care 2018; 34: e223-e225.

4. Acar Arslan E, Ekinci S, Zengin Akkuş P, Göçmen R, Haliloğlu G. Wernicke encephalopathy due to thiamine deficiency after surgery on a child with duodenal stenosis. Pediatr Neurol 2014; 51: 840-842.

5. Park SW, Yi YY, Han JW, Kim HD, Lee JS, Kang HC. Wernicke's encephalopathy in a child with high dose thiamine therapy. Korean J Pediatr 2014; 57: 496-499.

6. Cerase A, Rubenni E, Rufa A, et al. CT and MRI of Wernicke's encephalopathy. Radiol Med 2011; 116: 319-333.

7. Manzo G, Gennaro AD, Cozzolino A, Serino A, Fenza G, Manto A. MR imaging findings in alcoholic and nonalcoholic acute wernicke's encephalopathy: a review. Biomed Res Int 2014; 2014: 503596

8. Victor M, Adams RD, Collins GH. The WernickeKorsakoff syndrome: a clinical and pathological study of 245 patients, 82 with post-mortem examinations. Contemp Neurol Ser 1971; 7: 201-206. 
9. Manzo L, Locatelli C, Candura SM, Costa LG. Nutrition and alcohol neurotoxicity. Neurotoxicology 1994; 15: 555-565.

10. Harper CG, Giles M, Finlay-Jones R. Clinical signs in the Wernicke-Korsakoff complex: a retrospective analysis of 131 cases diagnosed at necropsy. J Neurol Neurosurg Psychiatry 1986; 49:341-345.

11. Vasconcelos MM, Silva KP, Vidal G, Silva AF, Domingues RC, Berditchevsky CR. Early diagnosis of pediatric Wernicke's encephalopathy. Pediatr Neurol 1999; 20: 289-294.

12. Lockman PR, McAfee JH, Geldenhuys WJ, Allen DD. Cation transport specificity at the blood-brain barrier. Neurochem Res 2004; 29: 2245-2250.

13. Chan F, Butterworth RF, Hazell AS. Primary cultures of rat astrocytes respond to thiamine deficiencyinduced swelling by downregulating aquaporin-4 levels. Neurosci Lett 2004; 366: 231-234.
14. Desjardins P, Butterworth RF. Role of mitochondrial dysfunction and oxidative stress in the pathogenesis of selective neuronal loss in Wernicke's encephalopathy. Mol Neurobiol 2005; 31: 17-25.

15. Mumford CJ. Papilloedema delaying diagnosis of Wernicke's encephalopathy in a comatose patient. Postgrad Med J 1989; 65: 371-373.

16. Manzanares W, Hardy G. Thiamine supplementation in the critically ill. Curr Opin Clin Nutr Metab Care 2011; 14: 610-617.

17. Zuccoli G, Pipitone N. MR Imaging: an increasingly important tool in the early diagnosis of Wernicke encephalopathy. AJNR Am J Neuroradiol 2012; 33: E92.

18. Galvin R, Brathen G, Ivashynka A, Hillbom M, Tanasescu R, Leone MA. EFNS guidelines for diagnosis, therapy and prevention of Wernicke encephalopathy. Eur J Neurol 2010; 17: 1408-1418. 